\title{
EVALUATION OF RECURRENT HOSPITALIZATION PROBABILITY IN PATIENTS WITH DUODENAL AND GASTRIC ULCER DEPENDENT UPON REHABILITATION INPUT
}

\author{
Oleksandr Ocheredko \\ Department of Public Health \\ Vinnitsa Pirogov Memorial National Medical Univertsity \\ 56 Pirogova str., Vinnitsa, Ukraine, 21018 \\ Ocheredko@yahoo.com \\ Natalia Kizlova \\ Department of Public Health \\ Vinnitsa Pirogov Memorial National Medical Univertsity \\ 56 Pirogova str., Vinnitsa, Ukraine, 21018
}

\begin{abstract}
Background: notoriously known worldwide cause of morbidity and disability duodenal (DU) and gastric ulcer (GU) experience their rise in Ukraine, demonstrating formidable increase by 38,4 \% in last decade with the prevalence of 2299 per 100000 population. Every second patient is treated in-patiently, every third experiences disability spell annually. Reduction in related risks confined not so much by absence of effective therapy but rather shortcomings in patient management and patient devotion. By WHO data $50 \%$ of patients fail to follow physician prescriptions, $60 \%$ can't recollect physician recommendations in first 20 minutes. Ubiquitous belated timing of rehabilitation initiation in post hospital stage appeared to be cardinal obstacle of its efficiency with low (up to $20 \%$ ) coverage, and ensuring clinical effect in $8 \%$ cases only.

Aim: to evaluate efficacy of rehabilitation program detailed at first episode of in-patient treatment at gastroenterological department.

Data: organized by cohort design. Control cohort comprised 180 patients with first episode of hospitalization due to DU or GU in gastroenterological Vinnitsa city department in 2009-2010 years. Experimental cohort consisted of 220 alike patients who enter rehabilitation program (RP). RP was administered randomly. Randomness was statistically verified on principal confounders. Cases were traced 4 years.

Methods: we applied three modifications of semi-parametric frailty model to study effect of program on the risk of recurrent hospitalization.

Results: all three modifications coincided in that program secured typically at least 39 days to recurrent hospitalization per patient with drop in risk at least at $\mathrm{RR}=0,774$.

Keywords: rehabilitation, duodenal and gastric ulcer, cohort design, frailty model.
\end{abstract}

\section{Introduction}

By the literature review and our experience timing of rehabilitation administration is the crucial to sustain its efficiency in terms of coverage, adherence, and clinical effect. Innovative to clinical experience in Ukraine is shift in administration of rehabilitation to hospital stage. We also worked out extended program frame that combines 10 scales, namely medication of ulcer, diet modification, overweight control, physiotherapy exercises, management of NSAID-induced gastropathy, risk factor management, blood pressure correction, diabetes management, anxiety and depression management. To empower compliance and to facilitate case management we supplied patient with diary and inculcate the skills of recording on drug intakes, unusual symptoms, complaints, as well as following indicated dates of examinations and physician referrals. The prime evidence of the efficacy of RP administration is the $4 \%$ of dropouts only in first 3 months $[1,2]$.

\section{Aim of research}

Evaluate efficacy of rehabilitation program detailed at first episode of in-patient treatment at gastroenterological department. 


\section{Materials and Methods}

Design. Data organized by cohort design. Control cohort comprised 180 patients with first episode of hospitalization with DU or GU diagnoses in gastroenterological Vinnitsa city department in 2009-2012 years. Experimental cohort consisted of 220 alike patients who enter RP. Program was adminstered randomly. Randomness was statistically verified on principal confounders $[3,4]$. Cases were traced for 4 years.

$R P$ frame. RP frame combines 10 scales, namely medication of ulcer, diet modification, overweight control, physiotherapy exercises, management of NSAID-induced gastropathy, risk factor management, blood pressure correction, diabetes management, anxiety and depression management. Each scale has its content, detailed explanation of administration, check points, efficacy evaluation. For instance, we lay out medication scale composition. Others in details brought out elsewhere $[5,6]$.

Medication scale. Content. Assessment of patient condition, fibrogastroscopia, intragastric pH monitoring, and H-pylori express diagnostic (De-Nol test) at hospitalization. Treatment of Hp-negative ulcers by antisecretory monotherapy starting with Group A1 - IPP of $1^{\text {st }}$ generation (omeprazole $40 \mathrm{mg}$ daily) during 4 weeks in case of duodenal ulcer and during 8 weeks in case of gastric ulcer. If prove to be ineffective in 10 days step to Group A2 - IPP of $3^{\text {rd }}$ line in standard dosage (rabeprazole $20 \mathrm{mg}$ daily), $4^{\text {th }}$ line in standard dosage (pantoprazole $40 \mathrm{mg}$ daily), $5^{\text {th }}$ line in standard dosage (ezomeprazole $40 \mathrm{mg}$ daily) during 4 weeks in case of duodenal ulcer and during 8 weeks in case of gastric ulcer. In persevering casesdouble dosage of $3^{\text {rd }}-5^{\text {th }}$ lines of IPP drugs applied. In Hp-positive cases eradication of H.pylori infection starts with B1 $-3^{\text {rd }}-5^{\text {th }}$ lines of IPP drugs (pantoprazole $20 \mathrm{mg}$ twice a day,rabeprazole $20 \mathrm{mg}$ twice a day, esomeprazole $20 \mathrm{mg}$ twice a day) + clarithromycin $500 \mathrm{mg}$ twice a day + amoxicillin $1000 \mathrm{mg}$ twice a day during 10 days and follows with administration of IPP drugs in standard dosages during 3 weeks in case of duodenal ulcer and during 6 weeks in case of gastric ulcer. If ineffective in 10 days step to B2 that is $3^{\text {rd }}-5^{\text {th }}$ lines of IPP drugs in standard dosages + tetracycline $500 \mathrm{mg} 4$ times a day + metronidazole $500 \mathrm{mg} 3$ times a day + bismuth subcitrate $120 \mathrm{mg} 4$ times a dayduring 10 days. Check points. Daily: self-control by indication of prescribed drugs intakes in diary. Weakly: assessment of clinical symptoms by general physician. Special points: evaluation of treatment efficacy at $10^{\text {th }}$ day intragastric $\mathrm{pH}$ monitoring in case of Hp-negative ulcers; evaluation ofH.pylori eradication in positive cases in two weeks upon treatment completion by fecal H.pylori antigen test; visits to gastroenterologist upon $1^{\text {st }}$ and $2^{\text {nd }}$ months. Efficacy evaluation. Patient follows prescribed therapy, admissible are 2 failures in day drug intake a month. Patient understands regimen of drug intakes. Achievement of clinical and endoscopic certified remission after 4 weeks of treatment: robust acid suppressive effect $(\mathrm{pH}>3)$ in 10 days from the start of treatment with IPP; successful H.pylori eradication in positive cases.

Follow up includes hospital and out of the hospital stages. To empower compliance and to facilitate case management we supplied patient with diary and inculcate the skills of recording on drug intakes, unusual symptoms, complaints, as well as following indicated dates of examinations and physician referrals. Diary proved to be helpful especially out of the hospital. The prime evidence of the efficacy of RP administration is the $4 \%$ of dropouts only in first 3 months.

We built efficacy estimation on time to next hospitalization that proved to be very sensitive to quality of care [7]. However, efficacy evaluation poses statistical challenge in part due to rana domization bias (e. g. self-selection bias), possible measurement error, or unavoidable presence of potent unobservable. So, treatment effect identification problem is conspicuous. We tackled it by control function technique $[7,8]$.

Model. We have chosen flexible semi-parametric frailty model to study modification effect of RP on the risk of recurrent hospitalization. Frailty model incapacitates the assessment of individual propensity to "survive" till next hospitalization incorporating unobserved patient's characteristic influenced risk of recurrent hospitalization differently across patients. Overlooking frailties entails biased and inefficient estimation of survival effects [9]. Frailty model basically incorporates three main components: basic hazard function, changeable in time; function of factors, modifying basic hazard; frailty distribution. Hazard function is defined non-parametrically by exponential piece-wisepriors [10]. The number of time intervals defined by 0,25 quantiles of observed time 
spans distribution that approximately coincides with monthly intervals. Pooling strength and identification of basic risks $\lambda_{\mathrm{j}}$ facilitated through RW1 process, namely (Win BUGS code):

$\operatorname{lam}[\mathrm{j}] \sim \operatorname{dgamma}(\mathrm{a} 0, \mathrm{~b} 0[\mathrm{j}])$

b0[j] <- a0/lam[j-1]

$\operatorname{lam}[1] \sim \operatorname{dgamma}(0.1,0.1)$,

$\mathrm{a} 0 \sim \operatorname{dgamma}(0.1,0.1)$

So that pooling is defined by gamma distribution with two first moments 0 and $\left(\lambda_{\mathrm{j}-1}\right)^{2} / \mathrm{a}_{0}$. Risk is defined as proportional to basic, modified by exponent of observable covariates effect $\beta_{j} \cdot x_{i}$ :

$$
h\left(t_{i} \in\left(q_{j-1}, q_{j}\right] \mid x_{i}\right)=\lambda_{j} \exp \left(\beta_{j} \cdot x_{i}\right),
$$

where $\beta_{\mathrm{j}} \cdot \mathrm{x}_{\mathrm{i}}$ is expressed by RP effect (parameter beta), bias in randomization of RP administration across patients (beta2), and individual random effects (frailties $b_{\mathrm{j}}$ ):

$$
\text { beta*Treatment }+ \text { beta } 2 * \text { Treatment } * b_{j}+b_{j} \text {. }
$$

The principal parameter to test ATE (average treatment effect) [11, 12] is beta coefficient, purged from possible randomization flaws of patient selection to RP prescription by present beta $2 *$ Treatment $* b_{j}$ component, that is the control function. $b_{j}$ render individual patient's effect with expected zero value, achieved by priors generation mechanism (rendered by $b[j] \sim d n o r m$ $(0$, tau $)$ in program script). Individual patient's effect includes all possible fixed individual effects both observable and unobservable. Presence of the latter is crucial for bias minimization in RP effect testing. Insignificant beta2 bares evidence on negligibility of bias in ATE estimation due to randomization flaws of patient selection to RP administration.

Cumulative risk (defined in programscript byH0) was calculated as integration of point risk $h\left(t_{i} \in\left(q_{j-1}, q_{j}\right] \mid x_{i}\right)$ on quantiles bounded time interval $q_{j}-q_{j-1}$ as follows:

$$
H 0_{j}=h\left(t \in\left(q_{j-1}, q_{j}\right] \mid x\right) *\left(q_{j}-q_{j-1}\right)=\lambda_{j} \exp \left(\beta_{j} \cdot x_{i}\right) *\left(q_{j}-q_{j-1}\right) .
$$

Survival functions in experimental and control cohorts members(defined in program code by $\mathrm{S}[1]$ and $\mathrm{S}[2]$ ) were calculated by formulas:

$$
\begin{aligned}
& \mathrm{S}[1]=\left[\exp \left(-\sum_{\mathrm{j}=1}^{40} \mathrm{H} 0_{\mathrm{j}}\right)\right]^{\exp (\text { beta })}, \\
& \mathrm{S}[2]=\exp \left(-\sum_{\mathrm{j}=1}^{40} \mathrm{H} 0_{\mathrm{j}}\right) .
\end{aligned}
$$

\section{Implementation and programming.}

Powerful modern tool to implement hierarchical mixt models proved to be MCMC modelling. We opted for convenient Gibbssampler. Programming performed in WinBUGS (Bayesian inference using Gibbs software) environment [13]. Data preparation as well as convergence diagnostics have been performed in environment of Rv.3.1.0 package CODA [14]. Displayed graphics were created by R package GRAPHICS. Program script is given below. It works in R environment. Script, data, initial values are passed to and processed by WinBUGS, activated through call «bugs» of $\mathrm{R}$ package R2WinBUGS.

Sampled values are returned to Rasspecial WinBUGS class object (named «results» in the script).

pkg<- «R2WinBUGS»

library $(\mathrm{pkg}$, character.only $=$ TRUE)

WD<-»C:/Dissertations/Natasha/NatashaGL5»

TD $<-\operatorname{getwd}()$

if(!is.null(WD) \& WD!=TD) setwd(WD)

data $<$-read.table $(«$ GastroStacked.txt», header $=$ TRUE $)$

model.file<- «Model/WinBugModel.txt» 
cat(«model \{

for (i in 1:I)

$\mathrm{TI}[\mathrm{i}]<-$ Treatment $[\mathrm{i}] * \mathrm{~b}[$ Patient $[\mathrm{i}]]$

for ( $\mathrm{k}$ in $1: \mathrm{K}-1$ ) \{\# risk status for subject $\mathrm{i}$ at interval $\mathrm{k}$,

$\mathrm{y}[\mathrm{i}, \mathrm{k}]<-\operatorname{step}($ Tbetw $[\mathrm{i}]-\mathrm{a}[\mathrm{k}]) * \operatorname{step}(\mathrm{a}[\mathrm{k}+1]$ - Tbetw[i])

\# times pentininterval $\mathrm{k}$

$\mathrm{o}[\mathrm{i}, \mathrm{k}]<-(\min (\text { Tbetw }[\mathrm{i}], \mathrm{a}[\mathrm{k}+1])-\mathrm{a}[\mathrm{k}])^{*} \operatorname{step}($ Tbetw $[\mathrm{i}]-\mathrm{a}[\mathrm{k}])$

\# piecewiseexponential

theta $[\mathrm{i}, \mathrm{k}]<-\operatorname{lam}[\mathrm{k}] * \exp ($ beta*Treatment $[\mathrm{i}]+$ beta $2 * \mathrm{TI}[\mathrm{i}]+\mathrm{b}[$ Patient [i]])

$\mathrm{mu}[\mathrm{i}, \mathrm{k}]<-\mathrm{o}[\mathrm{i}, \mathrm{k}] *$ theta $[\mathrm{i}, \mathrm{k}] ; \mathrm{y}[\mathrm{i}, \mathrm{k}] \sim \operatorname{dpois}(\mathrm{mu}[\mathrm{i}, \mathrm{k}])$;

\# likelihood (nuusedtoavoidlogsofzero)

$\mathrm{nu}[\mathrm{i}, \mathrm{k}]<-$ equals $(\mathrm{mu}[\mathrm{i}, \mathrm{k}], 0)+(1-\text { equals }(\mathrm{mu}[\mathrm{i}, \mathrm{k}], 0))^{*} \mathrm{mu}[\mathrm{i}, \mathrm{k}]$

$\mathrm{LL}[\mathrm{i}, \mathrm{k}]<-\mathrm{y}[\mathrm{i}, \mathrm{k}] * \log (\mathrm{nu}[\mathrm{i}, \mathrm{k}])-\mathrm{mu}[\mathrm{i}, \mathrm{k}]-\log \operatorname{fact}(\mathrm{y}[\mathrm{i}, \mathrm{k}])\}\}$

\# multi-levelvariation: Patienteffects

for $(\mathrm{j}$ in $1: \mathrm{NUM})\{\mathrm{b}[\mathrm{j}] \sim \operatorname{dnorm}(0, \mathrm{tau})$; b.r $[\mathrm{j}]<-\mathrm{b}[\mathrm{j}]-\operatorname{mean}(\mathrm{b}[])\}$

tau $\sim \operatorname{dgamma}(1,0.01)$

sig<- $1 /$ sqrt(tau)

\# Gammaprocesspriorsonbaselinehazard

for $(\mathrm{k}$ in $2: \mathrm{K}-1)\{\operatorname{lam}[\mathrm{k}] \sim \operatorname{dgamma}(\mathrm{a} 0, \mathrm{~b} 0[\mathrm{k}])$; b0[k] <- a0/lam[k-1]\}

$\operatorname{lam}[1] \sim \operatorname{dgamma}(0.1,0.1)$

\# treatmentparameter

beta $\sim \operatorname{dnorm}(0,0.001)$; beta2 $\sim \operatorname{dnorm}(0,0.001)$; a $0 \sim \operatorname{dgamma}(0.1,0.1)$

\# CumHazardandSurvivorship

$\mathrm{H} 0[1]<-\operatorname{lam}[1]^{*} \mathrm{a}[1]$; for $(\mathrm{k}$ in $2: \mathrm{K}-1)\left\{\mathrm{H} 0[\mathrm{k}]<-\operatorname{lam}[\mathrm{k}]^{*}(\mathrm{a}[\mathrm{k}]-\mathrm{a}[\mathrm{k}-1])\right\}$

for $(\mathrm{j}$ in $1: \mathrm{K}-1)\{\mathrm{S}[1, \mathrm{j}]<-\operatorname{pow}(\exp (-\operatorname{sum}(\mathrm{H} 0[1: \mathrm{j}])), \exp ($ beta $))$

$\mathrm{S}[2, \mathrm{j}]<-\exp (-\operatorname{sum}(\mathrm{H} 0[1: \mathrm{j}]))\}$

\# deviance

$\mathrm{Dv}<--2 * \operatorname{sum}(\mathrm{LL}[])\}$,$» , file=model.file )$

$\mathrm{q}<-$ quantile (data $\$$ Tbetw, probs $=\operatorname{seq}(0,100$, by $=2.5) / 100)$

$\mathrm{q}<$-as.numeric(q)

$\mathrm{K}<$-length(q)

I $<$ - nrow(data)

PatientNumber $<-400$

Tbetw $<$-data\$Tbetw

Treatment $<$-data\$Treatment

Patient $<$-data\$Patient

data $<-\operatorname{list}(\mathrm{a}=\mathrm{q}, \mathrm{K}=\mathrm{K}, \mathrm{I}=\mathrm{I}, \mathrm{NUM}=$ PatientNumber, Tbetw=Tbetw, Treatment=Treatment, Patient $=$ Patient)

inits $<$ - function ()\{

$\operatorname{list}(b=\operatorname{rep}(0$, times $=$ PatientNumber $), \operatorname{lam}=\operatorname{rep}(1$, times $=K-1)$, tau $=1, a 0=1$, beta $=0$, beta $2=0)\}$

parameters<- c («beta», «beta2», «lam», «sig», «a $0 »$, «S », «Dv»)

results $<-$ bugs $($ data $=$ data, inits $=$ inits, parameters.to.save $=$ parameters,

model=»WinBugModel.txt», debug=TRUE,

n.chains $=1$, n.iter $=10000$, bugs.seed $=1966$,

bugs.directory=»c:/Bugs/WinBUGS14»,

working.directory $=»$ Model»,

clearWD $=$ FALSE,

$\mathrm{DIC}=\mathrm{FALSE}$,

codaPkg=FALSE)

\#convergencydiagnostics: Geweke's,HeidelbergerandWelch'sconvergencediagnostictests: codat $<$-read.coda(output.file $=»$ Model $/$ codal.txt $\gg$, index.file $=»$ Model $/$ codaIndex.txt $)$ ) geweke $<$-geweke.diag(codat, frac1 $=0.1$, frac2 $=0.5$ )

$\mathrm{z}<$-geweke $\$ \mathrm{z}$ 


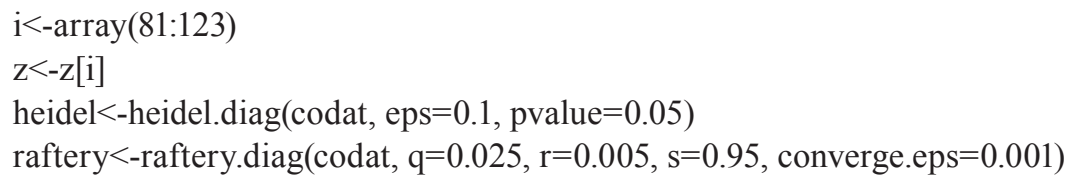

\section{Results}

Distribution of time spans (in days) to recurrent hospitalisation is displayed in Fig. 1. Distribution demonstrates right skew and conspiquous deviation from normality that in part calls for non-parametric approach [15]. Advantages of MCMC modelling also include capability of yielding posterior distributions of parameters of sampled values. We opted to display $5 \%(0,05)$ and $95 \%$ $(0,95)$ centiles of posterior distributions of sampled parameters values (Table 1) along with convergence diagnostics tests (Geweke's Zand Heidelberg -Welchhalfwidth value).

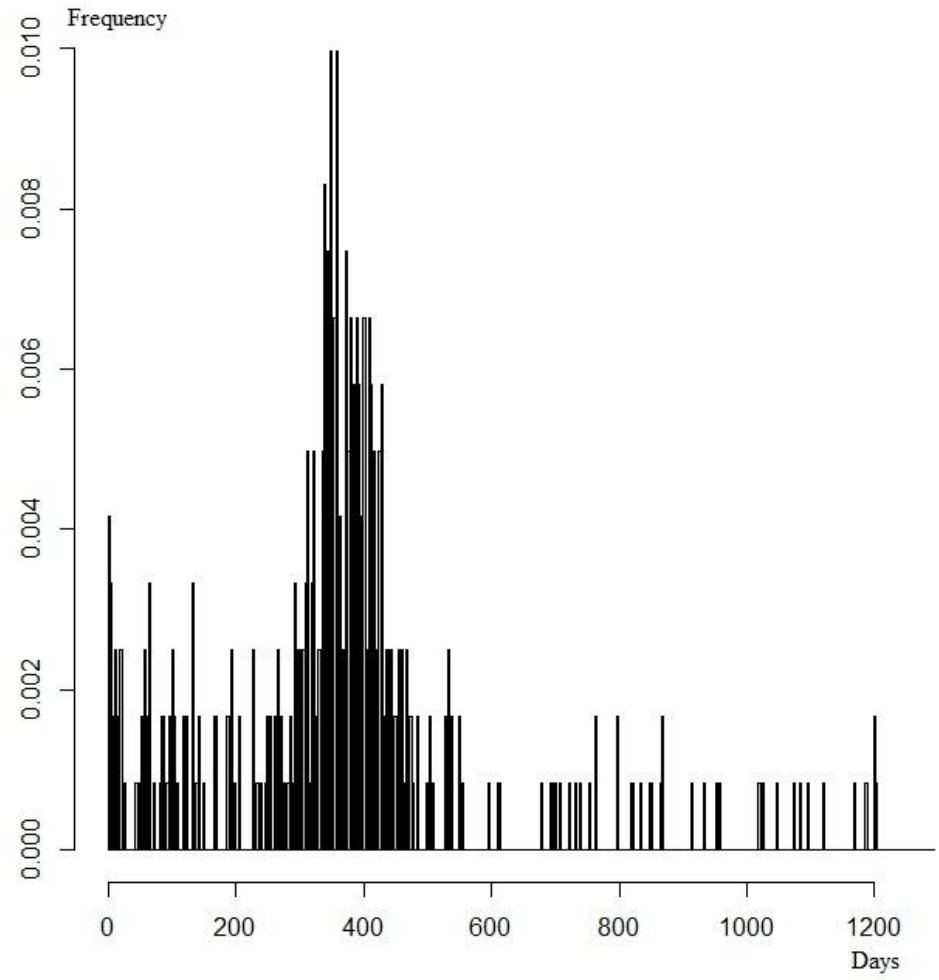

Fig. 1. Distribution of time spans to recurrent hospitalisation

Table 1

Centiles values of posterior distributions of sampled parameters values with convergence diagnostics tests

\begin{tabular}{cccccc}
\hline Parameters & $\mathbf{0 , 0 5}$ & $\begin{array}{c}\text { Centiles values } \\
\text { Median }\end{array}$ & $\mathbf{0 , 9 5}$ & \multicolumn{2}{c}{ Convergence tests } \\
& $-0,4010$ & $-0,2363$ & $-0,0701$ & 1,08 & H-W hl \\
\hline Beta & $-9,397$ & 0,8838 & 8,525 & $-0,12$ & 0,007660 \\
beta2 & 0,0009 & 0,0017 & 0,0028 & 0,78 & 0,012200 \\
$\lambda 1$ & 0,0008 & 0,0011 & 0,0016 & $-0,20$ & 0,000035 \\
$\lambda 2$ & 0,0009 & 0,0014 & 0,0021 & $-0,25$ & 0,000015 \\
$\lambda 3$ & & & & 0,000022 \\
& 0,0027 & 0,0039 & 0,0059 & $-1,84$ & 0,000071 \\
$\lambda 38$ & 0,0036 & 0,0054 & 0,0078 & $-1,05$ & 0,000169 \\
$\lambda 49$ & 0,0051 & 0,0081 & 0,0132 & $-1,71$ & 0,000251
\end{tabular}


The most important are parameters that actually estimate ATE of RP along with beta2 that makes allowance for possible randomization flaws of patient selection to RP and corrects for bias the ATE estimate due to heterogeneity of control and experimental cohorts correlated with selection. According to the results the ATE is proved to be significant leaving beyond the limits of $95 \%$ posterior distribution interval $[-0,4010 ;-0,0701]$ with median of $-0,2363$. Relative risk of RP administration on risk of recurrent hospitalization constituted $\exp (-0,2363)=0,790$. That is, risk of recurrent hospitalization reduced typically by $21 \%$ by RP administration.

Values of $5 \%(0,05)$ and $95 \%(0,95)$ centiles of posterior distribution of sampled beta 2 vali ues negate the significance of randomizationbias effect, for 0 lays in the middle of $95 \%$ posterior distribution interval which is $[-9,397 ; 8,525]$. Wide and symmetrical around posterior distribution $95 \%$ interval is conspicuous indication of the absence of randomization inconsistencies.We can suggest the absence of randomization induced bias in estimation of ATE that goes as corollary.

Median values of sampled values of basic risks of recurrent hospitalization on 40 time intert vals $(\lambda 1-\lambda 40)$ stipulated conspicuous pattern with rise and leveling off from 18 to 32 months from discharge followed by bluff decline (Fig. 2). All basic risk estimates proved to be significant for all $95 \%$ confidence intervals of posterior distributions left zero beyond.

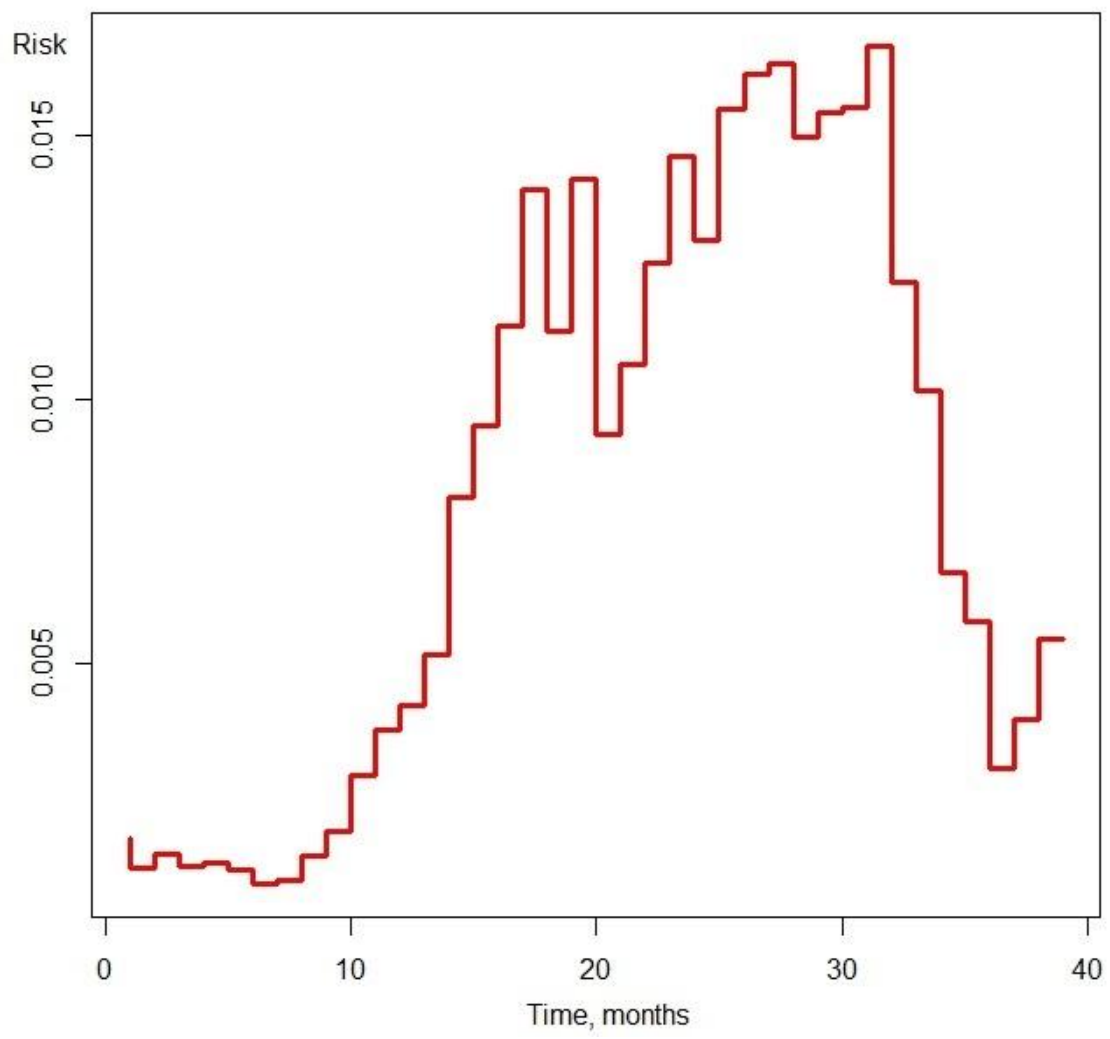

Fig. 2. Basic risks of recurrent hospitalization in 40 months

Two survival curves tracing proportions of patients in experimental and control cohorts in wait of recurrent hospitalization built by the model (Fig. 3).Distinct patterns were observed with decreased survival among control cohort representatives. Cumulated difference appeared to be 49 days per patient. This additional amount of days to next hospitalization safeguarded by RP that constitutes the effect of RP administration [16].

We also applied 2 extended models. Second one included important covariates in linear predictor. Only two covariates demonstrated marginally significant effect, namely GU with $95 \%$ confidence posterior interval of $-0,140-0,890$ and median 0,379 , that is increased risk of 1,46 against $\mathrm{DU}$, and regular visits to gastroenterologist with increased relative risk $\mathrm{RR}=1,24$. RP effect with $\mathrm{RR}=0,762$ saved 46 additional days to next hospitalization in average per patient. Still frailties 
distribution persisted to be heterogeneous that was tackled by third model that implemented scale mixture priors of frailties [5].

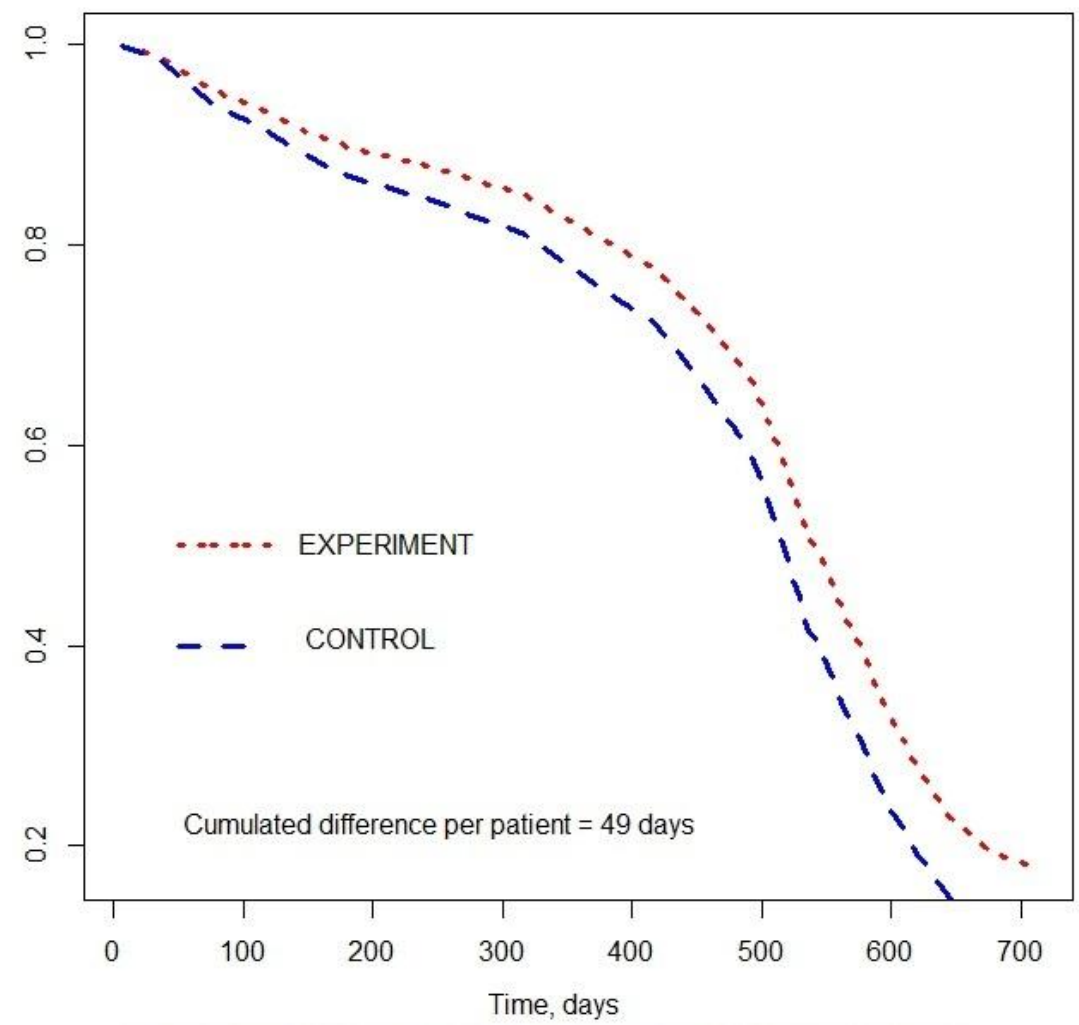

Fig. 3. Survival curves tracing proportions of patients in experimental and control cohorts in wait of recurrent hospitalization

The only significant fixed covariate effect rendered by contrast GU-DU. RP effect with $\mathrm{RR}=0,774$ saved 39 additional days. Obtained results and model comparison (Table 2) supported robustness of RP administration effect [17].

Table 2

Effects of RP administration by 3 models

\begin{tabular}{ccccc}
\hline Characteristics & Model \#1 & Model \#2 & Model \#3 & 3854 \\
Information value, $-2 \mathrm{LL}$ & 4095 & 4074 & 0,762 & 0,774 \\
RR & 0,790 & 46 & 39
\end{tabular}

\section{Discussion}

Evaluation of recurrent hospitalization probability in patients with duodenal and gastric ulcer dependent upon rehabilitation input proved RP efficacy in terms of risk reduction by RR of $0,77-0,79$. Given reduction secured 39-49 days to recurrent hospitalization. We rely on this measure of efficacy because episode of recurrent hospitalization is self-evident and uniformly registered, so measurement error, selectivity and reproducibility biases are minimized. Some may argues on economic efficiency assuming necessary increase in length of staying (LOS) and stipulating so the full policy implications of our analysis are mixed. Indeed, an increase inpatients' number of days in hospital is costly both in time and money, ceteris paribus. This is why a large number of health care policies such as the prospective payment system introduced in last decades mainly is aimed at reducing hospital LOS. On the other hand, such an increase may be partly justi- 
fied for two reasons. Firstly, it may be associated with more time spent by physicians on nonclinical activities such as teaching and administrative tasks, including rehabilitation efforts. Dumont, et. al., 2008 [18] provide evidence consistent with this effect as related to the Quebec reform. Second, as long as physicians spend more time treating their patients in hospital, this may improve patients' health. That is exactly suggested by this paper since the risk of re-hospitalization significantly and systematically decreased.

Our results raise an important issue regarding the measure of health care services quality in a given patients. Does the decrease in the risk of readmission to hospital necessarily indicate a better quality of services? We believe that this is the case still fretted with reservations. For instance, for a given diagnosis, physicians who spend more time with their patients in hospital to administer RP may also be more inclined to re-hospitalize them in order to provide them with a better treatment or follow-up [19, 20]. A natural research extension of our paper could thus be to compare the evolution of health status of two random groups of patients with a same diagnosis but one treated with RP administration and the other one without.

\section{Conclusions}

1. Belated timing of rehabilitation initiation in post hospital stage appeared to be cardinal obstacle of its efficiency with low (up to $20 \%$ ) coverage, and securing clinical effect in $8 \%$ cases only.

2. We shifted administration of rehabilitation to hospital stage. Program frame combines 10 scales, namely medication of ulcer, diet modification, overweight control, physiotherapy exercises, management of NSAID-induced gastropathy, risk factor management, blood pressure correction, diabetes management, anxiety and depression management.

3. To empower compliance and to facilitate case management we supplied patient with diary and inculcate the skills of recording on drug intakes, unusual symptoms, complaints, as well as following indicated dates of examinations and physician referrals. The prime evidence of the efficacy of RP administration is the $4 \%$ of dropouts only in first 3 months.

4. Program efficacy estimation relied upon time to recurrent hospitalization that proved to be very sensitive to quality of care. We have chosen flexible semi-parametric frailty models to study modification effect of program on the risk of recurrent hospitalization.

5. Results of three models coincided in that program secured typically at least 39 days to recurrent hospitalization per patient with drop in risk at least at $\mathrm{RR}=0,774$.

\section{References}

[1] Duchateau, L., Janssen, P. (2008). The Frailty Model. Springer, NewYork.

[2] Aalen, O. O., Hjort, N. L. (2002). Frailty models that yield proportional hazards. Statistics \& Probability Letters, 58 (4), 335-342. doi: 10.1016/s0167-7152(02)00090-1

[3] Ravishanker, N., Dey, D. (2000). Multivariate survival models with a mixture of positive stable frailties. Methodology and Computing in Applied Probability, 2 (3), 293-308. doi: 10.1023/a:1010033329399

[4] XXIV International Workshop on Helicobacter and related bacteria in chronic digestive inflammation and gastric cancer (2011). Dublin, Ireland.

[5] Aitkin, M., Clayton, D. (1980). The Fitting of Exponential, Weibull and Extreme Value Distributions to Complex Censored Survival Data Using GLIM. Applied Statistics, 29 (2), 156. doi: 10.2307/2986301

[6] Orbe, J., Núñez-Antón, V. (2006). Alternative approaches to study lifetime data under different scenarios: from the PH to the modified semiparametric AFT model. Computational Statistics \& Data Analysis, 50 (6), 1565-1582. doi: 10.1016/j.csda.2005.01.010

[7] Wooldridge, J. M. (2012). Control Function Methods in Econometrics. East Lansing, 45.

[8] Wooldridge, J. M. (2004). Estimating average partial effects under conditional moment independence assumptions. Institute for fiscal studies, 38.

[9] Therneau, T. M., Grambsch, P. M., Pankratz, V. S. (2003). Penalized Survival Models and Frailty. Journal of Computational and Graphical Statistics, 12 (1), 156-175. doi: 10.1198/1061860031365

[10] Littell, R. C., George, A. M., Walter, W. S., Russell, D. W., Oliver, S. (2006). SAS® for Mixed Models. Cary, NC: SAS Institute Inc., 834. 
[11] Congdon, P. D. (2010). Applied Bayesian Hierarchical Methods. Chapman and Hall/CRC, 604. doi: $10.1201 / 9781584887218$

[12] Rosenbaum, P. R., Rubin, D. B. (1983). The central role of the propensity score in observational studies for causal effects. Biometrika, 70 (1), 41-55. doi: 10.1093/biomet/70.1.41

[13] Lunn, D., Jackson, C., Best, N., Thomas, A., Spiegelhalter, D. (2012). The BUGS Book: A Practical Introduction to Bayesian Analysis. Chapman and Hall/CRC, 399.

[14] Torsten, H., Brian, S. E. (2014). A Handbook of Statistical Analyses Using R. Chapman \& Hall/ CRC Press, Boca Raton, Florida, USA.

[15] Altman, D. G., Bland, J. M. (2009). Parametric v non-parametric methods for data analysis. BMJ, 338, a3167-a3167. doi: 10.1136/bmj.a3167

[16] Ravishanker, N., Dey, D. (2015). Multivariate survival models with a mixture of positive stable frailties. Methodology and Computing in Applied Probability, 2, 293-308.

[17] Rice, K. (2005). Bayesian measures of goodness of fit. Encyclopedia of biostatistics. Chichester: John Wiley, 523. doi: 10.1002/0470011815.b2a09005

[18] Dumont, E., Fortin, B., Jacquemet, N., Shearer, B. (2008). Physicians' multitasking and incentives: Empirical evidence from a natural experiment. Journal of Health Economics, 27 (6), 1436-1450. doi: 10.1016/ j.jhealeco.2008.07.010

[19] Fo Fortin, B., Lacroix, G., Drolet, S. (2004). Welfare benefits and the duration of welfare spells: evidence from a natural experiment in Canada. Journal of Public Economics, 88 (7-8), 1495-1520. doi: 10.1016/ s0047-2727(02)00177-9

[20] Damien, É., Bernard, F. (2011). Physician Payment Mechanisms, Hospital Length of Stay and Risk of Readmission: a Natural Experiment. Version 1. 